CAS-KITPC/ITP-271 KIAS-P11036

\title{
Killing Spinors for the Bosonic String and the Kaluza-Klein Theory with Scalar Potentials
}

\author{
Haishan Liu ${ }^{1}$, H. L ̈̈ ${ }^{2,3}$ and Zhao-Long Wang ${ }^{4}$ \\ ${ }^{1}$ Zheijiang Institute of Modern Physics \\ Department of Physics, Zheijiang University, Hangzhou 310027, China \\ ${ }^{2}$ China Economics and Management Academy \\ Central University of Finance and Economics, Beijing 100081, China \\ ${ }^{3}$ Institute for Advanced Study, Shenzhen University \\ Nanhai Ave 3688, Shenzhen 518060, China \\ ${ }^{4}$ School of Physics, Korea Institute for Advanced Study, Seoul 130-722, Korea
}

\begin{abstract}
$\underline{\text { ABSTRACT }}$
The paper consists mainly of two parts. In the first part, we obtain well-defined Killing spinor equations for the low-energy effective action of the bosonic string with the conformal anomaly term. We show that the conformal anomaly term is the only scalar potential that one can add into the action that is consistent with the Killing spinor equations. In the second part, we demonstrate that the Kaluza-Klein theory can be gauged so that the Killing spinors are charged under the Kaluza-Klein vector. This gauging process generates a scalar potential with a maximum that gives rise to an AdS spacetime. We also construct solutions of these theories.
\end{abstract}




\section{Introduction}

An underlying property for the successful construction of supergravities is that the bosonic sector admits consistent Killing spinor equations, whose projected integrability condition gives rise to the full set of the bosonic equations of motion. This property exists in some intrinsically non-supersymmetric gravity theories as well. In fact, the concept of Killing spinor was introduced in Riemannian Geometry which predates the concept of supersymmetry. In pure Einstein gravities in diverse dimensions, which are not supersymmetric in general, a Killing spinor is defined to satisfy the Killing spinor equation $D_{M} \epsilon=0$. The integrability condition $\left[D_{M}, D_{N}\right] \epsilon=\frac{1}{4} R_{M N P Q} \Gamma^{P Q} \epsilon=0$ suggests that only a subset of Ricci-flat metrics may admit Killing spinors. However, the projected integrability condition, namely

$$
\Gamma^{M}\left[D_{M}, D_{N}\right] \epsilon=\frac{1}{2} R_{M N} \Gamma^{M} \epsilon=0,
$$

is satisfied automatically by the Einstein equation of motion. However, for generic gravity theories with scalar and form fields that cannot be supersymmetrized to become supergravities, consistent Killing spinor equations exist rarely.

Killing spinors for domain wall solutions in some scalar-gravity theories in general dimensions solutions were studied in [1], where the scalar potentials can be expressed in terms of superpotentials. We shall review this example and present the Killing spinor equations in section 2. A far more non-trivial example has recently been discovered that the low-energy effective action of the bosonic string in an arbitrary dimension admits Killing spinor equations [2, 3]. Furthermore, this property is up held even when arbitrary Yang-Mills fields are included, at least to the $\alpha^{\prime}$ quadratic curvature order [3]. This suggests that the hidden pseudo-supersymmetry associated with the existence of the Killing spinor equations is a stringy property, regardless whether the string is supersymmetric or not. It reflects some generalized geometric property of string theory.

One clue of constructing Killing spinor equations for the bosonic string is from the observation of the general study of pseudo-Killing spinor equations for Einstein gravity coupled to an $n$-form field strength [4]. By pseudo-Killing spinor equations, we mean that they are not consistent equations, in that the projected integrability conditions have extra constraints on the fields in additional to the equations of motion 1 Interestingly, there

\footnotetext{
${ }^{1}$ It should be emphasized that pseudo-Killing spinors can still be extremely useful in constructing nontrivial solutions including non-supersymmetric bubbling and less-bubbling AdS spaces, in which extra constraints can either be easily satisfied or give restrictions to the solution space [4. However, in this paper, we shall be concerned only with theories that admit the (consistent) Killing spinor equations.
} 
appears to be a mysterious connection between the consistency of Kaluza-Klein sphere reduction of a theory and the existence of Killing spinor equations. Since it was long established that it is consistent to perform the $S^{3}$ and $S^{D-3}$ reductions of the effective action of the bosonic string [5], Killing spinor equations were proposed in [2] and it was verified that their projected integrability conditions indeed give rise to the full set of the equations of motion, without any further constraint. The result was then generalized to include the $\alpha^{\prime}$ correction terms in [3].

However, in the previous discussions [2, 3, the conformal anomaly term arising from the bosonic string theory in the non-critical dimensions is set to zero. In section 3 , we construct Killing spinor equations with this term included. The construction can be expected to be successful since this term has been shown not to spoil the consistency of the sphere reduction [5]. In fact, we consider a general scalar potential that can be expressed in terms of a generic superpotential. Although Killing spinor equations exist for either the bosonic string without the scalar potential, or the pure scalar-gravity system, the projected integrability conditions of the full system give rise to extra conditions that fix the scalar potential. It turns out that the conformal anomaly term and only this scalar potential works with the Killing spinor equations. We also find that the system can be pushed to include the $\alpha^{\prime}$-order corrections as well.

Killing spinor equations also exist in the Kaluza-Klein theory that is the $S^{1}$ reduction of pure gravity [2]. In section 4, we consider adding a scalar potential. We find that as in the case of the string theory, the projected integrability of the Killing spinor equations restricts the scalar potential to a single particular exponential term, analogous to the conformal anomaly term in string theory. We compare the results with the $S^{1}$ reduction of pure gravity with a cosmological constant in the appendix and find that these two scalar potentials are different.

The existence of a vector field in Kaluza-Klein theory provides a possibility of gauging the theory such that the Killing spinors are charged under the gauge symmetry. In section 5 , we demonstrate that the effect of the gauging is that both the superpotential and the scalar potential contain additional exponential scalar factors. The (super) potential has a maximum that gives rise to the AdS spacetime. This gauged Kaluza-Klein theory can be embedded in known gauged supergravities in four, five and seven dimensions. We obtain the charged AdS black hole and discuss its properties.

One advantage of having Killing spinor equations for a pure bosonic system is that it may help to construct a general class of BPS solutions that preserve Killing spinors. Some 
of these solutions are unlikely to be found by trying to solve the bosonic equations of motion alone. In section 6 , we use the $D=5$ string theory as an example to demonstrate this point and obtain the most general solutions involving Killing spinors. We conclude the the paper in section 7 .

\section{Scalar-gravity theory}

In supergravities, there are two types of solutions. Those that admit Killing spniors are called BPS solutions. These solutions preserve certain fractions of the supersymmetry of the theories. The other type of solutions are non-supersymmetric. However, it was observed that even the Schwarzschild-AdS black hole can be solved by the super-potential method in which the second-order differential equations can be successfully reduced to a set of first-order equations via a super potential [6, 7]. (See also [8].) This suggests that certain non-supersymmetric systems may exhibit characteristics of supersymmetry; they are pseudo-supersymmetric. Pseudo-supersymmetry for de Sitter "supergravity" was discussed in [9, 10]. Note that, owing to the lack of clear definition, the concept of pseudosupersymmetry in the past literatures may refer both solutions and abstract theories.

In this section, We consider gravity coupled to a scalar with a scalar potential in $D$ dimensions. The Lagrangian is given by

$$
\mathcal{L}_{D}=\sqrt{-g}\left(R-\frac{1}{2}(\partial \phi)^{2}-V(\phi)\right)
$$

We now determine the condition for $V$ such that the above system admits consistent Killing spinor equations. Inspired by supergravities, we introduce a super potential $W(\phi)$ in the Killing spinor equations, which are given by

$$
D_{M} \eta+\frac{W}{2 \sqrt{2}(D-2)} \Gamma_{M} \eta=0, \quad \Gamma^{M} \partial_{M} \phi \eta-\sqrt{2} W^{\prime} \eta=0
$$

where a prime denotes a derivative with respect to $\phi$. In the above equations, we start with two arbitrary coefficients for the $W$ and $W^{\prime}$ terms. The consistency of the projected integrability condition fixes the above two coefficients. Furthermore, it relates the potential $V$ to the super potential $W$ as follows

$$
V=\left(\frac{d W}{d \phi}\right)^{2}-\frac{D-1}{2(D-2)} W^{2}
$$

The projected integrability conditions are then given by

$$
\left(R_{M N}-\frac{1}{2} \partial_{M} \phi \partial_{N} \phi-\frac{V}{D-2} g_{M N}\right) \Gamma^{M} \eta=0, \quad\left(\square \phi-V^{\prime}\right) \eta=0 .
$$


Thus provided that the potential $V$ can be expressed in terms of a superpotential $W$, the scalar-gravity theory has well-defined Killing spinor equations. The Killing spinor equations for such a scalar-gravity theory were studied in the context of AdS domain wall solutions [1. The application of the scalar-gravity theory in cosmology was discussed in [11].

\section{Bosonic string with the conformal anomaly term}

The critical dimension for the bosonic string is $D=26$. It suffers from a conformal anomaly in the dimension $D \neq 26$. It turns out that the effect of this anomaly is to generate an additional scalar potential of the dilaton to the effective action [12]

$$
\mathcal{L}_{D}=\sqrt{-g}\left(R-\frac{1}{2}(\partial \phi)^{2}-\frac{1}{12} e^{a \phi} H_{(3)}^{2}-V\right)
$$

where $H_{(3)}=d B_{(2)}, a=2 \sqrt{2} /(D-2)$ and

$$
V=\frac{1}{2}(D-26) m^{2} e^{-\frac{1}{2} a \phi} .
$$

It was shown that it is consistent to perform $S^{3}$ and $S^{D-3}$ reductions even when the conformal anomaly term is present [5]. In the absence of the conformal anomaly term, the Killing spinor equations for this system were obtained in [2, 3]. The ansatz for the Killing spinor equations with the anomaly term is

$$
\begin{aligned}
D_{M} \eta+\frac{1}{96} e^{\frac{1}{2} a \phi}\left(a^{2} \Gamma_{M} \Gamma^{N P Q}-12 \delta_{M}^{N} \Gamma^{P Q}\right) H_{N P Q} \eta+\frac{W}{2 \sqrt{2}(D-2)} \Gamma_{M} \eta & =0 \\
\left(\Gamma^{M} \partial_{M} \phi+\frac{1}{12} a e^{\frac{1}{2} a \phi} \Gamma^{M N P} H_{M N P}-\sqrt{2} W^{\prime}\right) \eta & =0,
\end{aligned}
$$

As we see in section 2, one necessary condition for the above equation to be consistent is that the scalar potential $V$ and its superpotential $W$ must be related by (4). Thus, given that the scalar potential $V$ is known (7), $W$ can be straightforwardly determined. However, it is still necessary to check the full projected integrability conditions to verify whether they are consistent with equations of motion. Furthermore, one may also ask whether the theory (6) can admit Killing spinor equations for other scalar superpotentials.

Thus for now, let us consider that $V$ is given by (44), but with the superpotential $W$ being unspecified. We shall use the consistency of the projected integrability condition to determine the superpotential $W$ and hence the potential $V$.

Acting on (9) with $\Gamma^{N} D_{N}$, we find that

$$
\left(\nabla^{2} \phi-\frac{1}{12} a e^{a \phi} H^{2}-V^{\prime}(\phi)\right) \eta+\frac{1}{12} a e^{\frac{1}{2} a \phi} \Gamma^{N M_{1} M_{2} M_{3}} \nabla_{N} H_{M_{1} M_{2} M_{3}} \eta
$$




$$
+\frac{1}{4} a e^{-\frac{1}{2} a \phi} \Gamma^{M_{2} M_{3}} \nabla_{N}\left(e^{a \phi} H^{N}{ }_{M_{2} M_{3}}\right) \eta-U_{1} e^{\frac{1}{2} a \phi} \Gamma^{M_{1} M_{2} M_{3}} H_{M_{1} M_{2} M_{3}} \eta=0,
$$

where $V$ is given by (44) and

$$
U_{1}=\frac{1}{3 \sqrt{D-2}}\left(W^{\prime \prime}-\frac{1}{\sqrt{2(D-2)}} W^{\prime}-\frac{1}{D-2} W\right) .
$$

Thus for the projected integrability condition to be consistent with the equations of motion, we must have $U_{1}=0$, implying that

$$
W=c_{1} e^{-\frac{\phi}{\sqrt{2(D-2)}}}+c_{2} e^{\frac{2 \phi}{\sqrt{2(D-2)}}} .
$$

We now examine the projected integrability condition for (8). we find

$$
\begin{aligned}
& {\left[R_{M N}-\frac{1}{2} \partial_{M} \phi \partial_{N} \phi-\frac{1}{4} e^{a \phi}\left(H_{M N}^{2}-\frac{2}{3(D-2)} H^{2} g_{M N}\right)-\frac{V}{D-2} g_{M N}\right] \Gamma^{N} \eta} \\
& -\frac{1}{6(D-2)} e^{\frac{1}{2} a \phi} \nabla_{N} H_{M_{1} M_{2} M_{3}}\left(\Gamma_{M} \Gamma^{N M_{1} M_{2} M_{3}}-2(D-2) \delta_{M}^{[N} \Gamma^{\left.M_{1} M_{2} M_{3}\right]}\right) \eta \\
& -\frac{1}{2(D-2)} e^{-\frac{1}{2} a \phi} \nabla_{N}\left(e^{a \phi} H^{N} M_{2} M_{3}\right)\left(\Gamma_{M} \Gamma^{M_{2} M_{3}}-(D-2) \delta_{M}^{M_{2}} \Gamma^{M_{3}}\right) \eta \\
& +\frac{1}{4(D-2)^{\frac{3}{2}}} U_{2} e^{\frac{1}{2} a \phi}\left(\Gamma_{M} \Gamma^{M_{1} M_{2} M_{3}}-(D-2) \delta_{M}^{M_{1}} \Gamma^{M_{2} M_{3}}\right) H_{M_{1} M_{2} M_{3}} \eta=0,
\end{aligned}
$$

where

$$
U_{2}=W^{\prime}+\frac{1}{\sqrt{2(D-2)}} W
$$

It is easy to see that the solution (12) with $c_{2}=0$ satisfy the equation $U_{2}=0$. Thus, the scalar potential is precisely the conformal anomaly term provided that the non-vanishing constant $c_{2}$ is given by

$$
c_{1}=\sqrt{26-D} m
$$

Thus we demonstrate that the presence of the conformal anomaly term does not spoil the existence of the consistent Killing spinor equations. Furthermore, the conformal anomaly term is the only scalar potential that we can add to the bosonic string such that Killing spinor equations remain consistent. The result confirms the suggestion that there is an underlying generalized geometric structure associated with Killing spnior equations in string theory, whether it is supersymmetric or not, critical or non-critical.

In the string frame, defined by $d s_{\text {string }}^{2}=e^{-\frac{1}{2} a \phi} d s_{\text {Einstein }}^{2}$, the effective Lagrangian becomes

$$
\mathcal{L}=\sqrt{-g} e^{-2 \Phi}\left(R+4(\partial \Phi)^{2}-\frac{1}{12} H_{(3)}^{2}+\frac{1}{2} c_{1}^{2}\right),
$$

where $\Phi=-\phi / a$. The Killing spinor equations, after scaling $\eta \rightarrow e^{-\frac{1}{8} a \phi} \eta$, become

$$
D_{M}\left(\omega_{-}\right) \eta=0, \quad\left(\Gamma^{M} \partial_{M} \Phi-\frac{1}{12} \Gamma^{M N P} H_{M N P}+\frac{c_{1}}{2 \sqrt{2}}\right) \eta=0
$$


where $\omega_{-}$is the torsionful spin connection, given by

$$
\omega_{M \pm}^{A B}=\omega_{M}^{A B} \pm \frac{1}{2} H_{M}^{A B}
$$

It is interesting to note that the equation $D_{M}\left(\omega_{-}\right) \eta=0$ is unmodified by the conformal anomaly.

It was shown in [3] that the Killing spinor equations can be well-defined for the effective action of the bosonic string with arbitrary Yang-Mills fields up to the $\alpha^{\prime}$ order. A crucial property that enables one to write the action up to the quadratic curvature terms is the first equation in (17) [3]. This equation is unmodified by the conformal anomaly. This implies that the procedure of obtaining the $\alpha^{\prime}$-order Lagrangian works equally well with the presence of the conformal anomaly. We find that the most general Lagrangian at the tree level, up to the $\alpha^{\prime}$ order, is given by

$$
\mathcal{L}_{D}=\sqrt{-g} e^{-2 \Phi}\left[R+\frac{1}{2} c_{1}^{2}+4(\partial \phi)^{2}-\frac{1}{12} H_{(3)}^{2}-\frac{1}{4} \alpha\left(\operatorname{tr}^{\prime} F_{(2)}^{2}-R_{M N A B}\left(\omega_{+}\right) R^{M N A B}\left(\omega_{+}\right)\right)\right],
$$

where

$$
d H_{(3)}=\frac{1}{2} \alpha\left(\operatorname{tr}\left(R_{(2)}\left(\omega_{+}\right) \wedge R_{(2)}\left(\omega_{+}\right)\right)-\operatorname{tr}^{\prime}\left(F_{(2)} \wedge F_{(2)}\right)\right) .
$$

The Killing spinors equations are given by

$$
D_{M}\left(\omega_{-}\right) \eta=0, \quad\left(\Gamma^{M} \partial_{M} \Phi-\frac{1}{12} \Gamma^{M N P} H_{M N P}+\frac{c_{1}}{2 \sqrt{2}}\right) \eta=0, \quad \Gamma^{M_{1} M_{2}} F_{M_{1} M_{2}} \eta=0,
$$

We adopt exactly the same notation as in [3].

\section{Kaluza-Klein theory with a scalar potential}

In this section we consider Kaluza-Klein theory with a scalar potential

$$
\mathcal{L}_{D}=\sqrt{-g}\left(R-\frac{1}{2}(\partial \phi)^{2}-\frac{1}{4} e^{a \phi} F_{(2)}^{2}-V(\phi)\right),
$$

where $F_{(2)}=d A_{(1)}, a=\sqrt{2(D-1) /(D-2)}$. For $V=0$, the Lagrangian is the $S^{1}$ reduction of $(D+1)$-dimensional pure gravity, and $A_{(1)}$ is the Kaluza-Klein vector. It was shown in [2] that the system admits well-defined Killing spinor equations. Following the discussion of the bosonic string theory with the conformal anomaly, we now derive the scalar potential $V$ such that consistent Killing spinor equations can still be defined. As discussed in section 2, a necessary condition is that $V$ can be expressed in terms of a superpotential $W$ as in (41). The ansatz for the Killing spinor equations is

$$
D_{M} \eta+\frac{\mathrm{i}}{8(D-2)} e^{\frac{1}{2} a \phi}\left(\Gamma_{M} \Gamma^{M_{1} M_{2}}-2(D-2) \delta_{M}^{M_{1}} \Gamma^{M_{2}}\right) F_{M_{1} M_{2}} \eta
$$




$$
\begin{gathered}
+\frac{W}{2 \sqrt{2}(D-2)} \Gamma_{M} \eta=0, \\
\Gamma^{M} \partial_{M} \phi \eta+\frac{\mathrm{i}}{4} a e^{\frac{1}{2} a \phi} \Gamma^{M_{1} M_{2}} F_{M_{1} M_{2}} \eta-\sqrt{2} \frac{d W}{d \phi} \eta=0 .
\end{gathered}
$$

We find that the projected integrability condition for the second equation in (23) is given by

$$
\begin{aligned}
& \left(\nabla^{2} \phi-\frac{1}{4} a e^{a \phi} F^{2}-V^{\prime}(\phi)\right) \eta+\frac{\mathrm{i}}{4} a e^{\frac{1}{2} a \phi} \Gamma^{N M_{1} M_{2}} \nabla_{N} F_{M_{1} M_{2}} \eta \\
& +\frac{\mathrm{i}}{2} a e^{-\frac{1}{2} a \phi} \Gamma^{M_{2}} \nabla_{N}\left(e^{a \phi} F_{M_{2}}^{N}\right) \eta-\frac{\mathrm{i}}{2} \sqrt{\frac{D-1}{D-2}} U_{1} e^{\frac{1}{2} a \phi} \Gamma^{M_{1} M_{2}} F_{M_{1} M_{2}} \eta=0,
\end{aligned}
$$

where $U_{1}$ is a function of $W, W^{\prime}$ and $W^{\prime \prime}$, given by

$$
U_{1}=W^{\prime \prime}+\sqrt{\frac{2}{(D-1)(D-2)}} W^{\prime}-\frac{D-3}{2(D-2)} W .
$$

Since the structure $U_{1}$ is unrelated to any of the equations of motion, it has to vanish on its own. The solution to $U_{1}=0$ is

$$
W=c_{1} e^{\frac{D-3}{\sqrt{2(D-1)(D-2)}} \phi}+c_{2} e^{-\frac{D-1}{\sqrt{2(D-1)(D-2)}} \phi} .
$$

The projected integrability condition for the first equation of (23) is given by

$$
\begin{aligned}
& {\left[R_{M N}-\frac{1}{2} \partial_{M} \phi \partial_{N} \phi-\frac{1}{2} e^{a \phi}\left(F_{M N}^{2}-\frac{1}{2(D-2)} F^{2} g_{M N}\right)-\frac{1}{D-2} V(\phi) g_{M N}\right] \Gamma^{N} \eta} \\
& -\frac{\mathrm{i}}{4(D-2)} e^{\frac{1}{2} a \phi} \nabla_{N} F_{M_{1} M_{2}}\left(\Gamma_{M} \Gamma^{N M_{1} M_{2}}-3(D-2) \delta_{M}^{[N} \Gamma^{\left.M_{1} M_{2}\right]}\right) \eta \\
& -\frac{\mathrm{i}}{2(D-2)} e^{-\frac{1}{2} a \phi} \nabla_{N}\left(e^{a \phi} F^{N} M_{2}\right)\left(\Gamma_{M} \Gamma^{M_{2}}-(D-2) \delta_{M}^{M_{2}}\right) \eta \\
& +U_{2} \delta_{M}^{M_{1}} \Gamma^{M_{2}} e^{\frac{1}{2} a \phi} F_{M_{1} M_{2}} \eta=0
\end{aligned}
$$

where

$$
U_{2}=\frac{\mathrm{i}}{2} \sqrt{\frac{D-1}{D-2}} W^{\prime}-\frac{\mathrm{i} \sqrt{2}(\mathrm{D}-3)}{4(D-2)} W .
$$

Again the term $U_{2}$ is unrelated to any of the equations of motion and it has to vanish. However, it is easy to see that $W$ in (26) with $c_{2}=0$ satisfy the equation $U_{2}=0$. Thus the scalar potential $V$ is given by

$$
V=-\frac{2 c_{1}^{2}}{D-1} e^{\frac{2(D-3)}{\sqrt{2(D-1)(D-2)}} \phi} .
$$

Naively one would expect that the this single exponential scalar potential has an origin as the cosmological constant in Einstein gravity in $D+1$ dimensions, since cosmological Einstein gravity does accept a well-defined Killing spinor equation. However, as we show in the appendix, the scalar potential from $S^{1}$ reduction of the cosmological constant, given by (69), is different from this structure. Thus the origin of this scalar potential remains to be understood. 


\section{Gauging the Killing spinors and AdS spacetimes}

With the Kaluza-Klein vector, it is possible that we can gauge the theory such that fermions are charged under the vector. In the case of supergravities, this procedure may turn a supergravity theory to the gauged supergravity, where a scalar potential is generated. Let us propose that the Killing spinors are charged under $A_{(1)}$. The equations are now given by

$$
\begin{gathered}
\left(D_{M}+b A_{M}\right) \eta+\frac{\mathrm{i}}{8(D-2)} e^{\frac{1}{2} a \phi}\left(\Gamma_{M} \Gamma^{M_{1} M_{2}}-2(D-2) \delta_{M}^{M_{1}} \Gamma^{M_{2}}\right) F_{M_{1} M_{2}} \eta \\
+\frac{W}{2 \sqrt{2}(D-2)} \Gamma_{M} \eta=0, \\
\Gamma^{M} \partial_{M} \phi \eta+\frac{\mathrm{i}}{4} a e^{\frac{1}{2} a \phi} \Gamma^{M_{1} M_{2}} F_{M_{1} M_{2}} \eta-\sqrt{2} \frac{d W}{d \phi} \eta=0,
\end{gathered}
$$

where the constant $b$ is to be determined. Note that these equations (30) are invariant under the gauge transformation

$$
A_{(1)} \rightarrow A_{(1)}+d \Lambda, \quad \eta \rightarrow \eta e^{-b \Lambda}
$$

The projected integrability condition for the second equation in (30) remains the same as the previous ungauged case. It implies that $W$ is given by (26). Note that we can shift $\phi$ by constant to adjust the relative coefficients of $c_{1}$ and $c_{2}$. Make a convention that the fixed point occurs at $\phi=0$, we have

$$
c_{1}=\frac{D-1}{\sqrt{2}} g, \quad c_{2}=\frac{D-3}{\sqrt{2}} g .
$$

We have chosen the convention such that $g^{2}=1$ corresponds to the AdS with unit length. The projected integrability condition for the first equation in (30) gives the same form as (27), but now with $U_{2}$ given by

$$
U_{2}=\frac{\mathrm{i}}{2} \sqrt{\frac{D-1}{D-2}} W^{\prime}-\frac{\mathrm{i} \sqrt{2}(\mathrm{D}-3)}{4(D-2)} W+2 b e^{-\frac{1}{2} a \phi} .
$$

Thus the vanishing of $U_{2}$ implies that

$$
b=-\mathrm{i} \frac{\sqrt{2}}{4} c_{2}=-\frac{\mathrm{i}}{4}(D-3) g
$$

The full consistent Killing spinor equations are now given by

$$
\begin{aligned}
& \left(D_{M}-\frac{1}{4}(D-3) \text { i } g A_{M}\right) \eta+\frac{W}{2 \sqrt{2}(D-2)} \Gamma_{M} \eta \\
& \quad+\frac{\mathrm{i}}{8(D-2)} e^{\frac{1}{2} a \phi}\left(\Gamma_{M} \Gamma^{M_{1} M_{2}}-2(D-2) \delta_{M}^{M_{1}} \Gamma^{M_{2}}\right) F_{M_{1} M_{2}} \eta=0 \\
& \Gamma^{M} \partial_{M} \phi \eta+\frac{\mathrm{i}}{4} a e^{\frac{1}{2} a \phi} \Gamma^{M_{1} M_{2}} F_{M_{1} M_{2}} \eta-\sqrt{2} \frac{d W}{d \phi} \eta=0,
\end{aligned}
$$


where the super potential $W$ is completely determined, given by

$$
W=\frac{g}{\sqrt{2}}\left((D-3) e^{-\frac{D-1}{\sqrt{2(D-1)(D-2)}} \phi}+(D-1) e^{\frac{D-3}{\sqrt{2(D-1)(D-2)}} \phi}\right) .
$$

The corresponding scalar potential is

$$
V=-g^{2}(D-1)\left((D-3) e^{-\sqrt{\frac{2}{(D-1)(D-2)}} \phi}+e^{\frac{\sqrt{2}(D-3)}{\sqrt{(D-1)(D-2)}} \phi}\right) .
$$

It is clear that this potential has a maximum at $\phi=0$ with $V(0)=-(D-1)(D-2) g^{2}$.

We find that the Lagrangian (22) with the scalar potential (37) can be embedded in gauged supergravities in $D=4,5$ and 7 . (See, for example, [13.) In the case of $D=6$, it may also be possible to embed the theory in the $F(4)$ gauged supergraity [14] coupled to a vector multiplet [15, 16]. Thus it may not be surprising that the Kaluza-Klein theories with this scalar potential in $D=4,5,6$ and 7 admit Killing spinor equations. However it is of great interest to observe that the theory can admit Killing spinor equations in an arbitrary dimnension $D$.

We obtain the charged black hole solutions in general dimensions, for this gauged KaluzaKlein theory; they are given by

$$
\begin{aligned}
& d s_{D}^{2}=-H^{-\frac{D-3}{D-2}} f d t^{2}+H^{\frac{1}{D-2}}\left(\frac{d r^{2}}{f}+r^{2} d \Omega_{(D-2), k}^{2}\right), \\
& F_{(2)}=\sqrt{k} \operatorname{coth} \delta d t \wedge d H^{-1}, \quad \phi=\frac{1}{2} a \log H
\end{aligned}
$$

where

$$
H=1+\frac{\mu \sinh ^{2} \delta}{k r^{D-3}}, \quad f=k-\frac{\mu}{r^{D-3}}+g^{2} r^{2} H,
$$

and $k=1,0,-1$ corresponding $d \Omega_{(D-2), k}^{2}$ being the sphere, torus and hyperbolic spaces. In the case of $k=0$, we need to scale $\sinh ^{2} \delta \rightarrow k \sinh ^{2} \delta$ before sending $k$ to zero, and hence $F_{(2)}$ for $k=0$ is given by

$$
F_{(2)}=\frac{1}{\sinh \delta} d t \wedge d H^{-1}
$$

These solutions in relevant gauged supergravities in $D=4,5,6$ and 7 have string and M-theory origins [13].

We now examine the thermodynamical properties of the black hole (38), with $k=1$. The temperature, entropy, electric potential and charge are given by

$$
\begin{aligned}
T & =\left.\frac{f^{\prime}(r)}{4 \pi H^{1 / 2}}\right|_{r=r_{+}}, \quad S=\frac{1}{4} H^{1 / 2} r_{+}^{D-2} \omega_{D-2}, \\
\Phi & =\left(1-H^{-1}\right) \operatorname{coth} \delta, \quad Q=\frac{D-3}{32 \pi} \mu \sinh (2 \delta) \omega_{D-2} .
\end{aligned}
$$


The mass of the black hole is given by

$$
M=\frac{r_{+}^{D-3}\left(1+g^{2} r_{+}^{2}\right)(D-1+(D-3) \cosh (2 \delta))}{32 \pi\left(1-g^{2} r_{+}^{2} \sinh ^{2} \delta\right)} .
$$

The BPS limit is given by setting $\mu \rightarrow 0$ while keeping $\mu \sinh ^{2} \delta=q$ fixed, for which $H=1+q / r^{D-3}$ and $f=1+g^{2} r^{2} H$ and $\operatorname{coth} \delta=1$. The Killing spinors exist in this BPS limit, satisfying the following projection

$$
\left(f^{\frac{1}{2}}+\mathrm{i} \Gamma^{t}+g r H^{\frac{1}{2}} \Gamma^{r}\right) \eta=0
$$

For $k=0$, the extremal limit implies the vanishing of the 2 -form and the resulting solution is the BPS domain wall.

To conclude this section, we would like to mention that the general charged rotating black holes in the Kaluza-Klein theory with the scalar potential (37) was obtained in [18.

\section{A class of general "BPS" solutions}

The existence of well-defined Killing spinor equations can be a powerful tool in finding "BPS" solutions that preserve at least one Killing spinor [17]. As an example, let us consider the effective action of the bosonic string at $D=5$, with the conformal anomaly term set to 0 . The Killing spinor equations are given by (8) and (9), with $W=0=W^{\prime}$. In $D=5$, the spinors are pseudo-Majorana, and hence all possible bi-spinors take the following form

$$
f=\mathrm{i} \bar{\eta} \eta, \quad K^{M}=\bar{\eta} \Gamma^{M} \eta, \quad Y_{(3)}^{M N}=\bar{\eta} \Gamma^{M N} \eta,
$$

which are real, together with

$$
Y^{M N}=\bar{\eta}^{c} \Gamma^{M N} \eta \equiv Y_{(1)}^{M N}+i Y_{(2)}^{M N}
$$

which is complex. Here, we have $\bar{\eta}=\eta^{\dagger} \Gamma_{0}$ and $\bar{\eta}^{c}=\eta^{T} C$, where $C$ is the charge conjugation matrix. We find the following identity for these bi-spinors:

$$
\begin{aligned}
& \nabla_{M} f=\frac{1}{18} e^{\frac{1}{2} a \phi} \epsilon_{M}^{M_{1} M_{2} M_{3} N} H_{M_{1} M_{2} M_{3} K_{N},} \\
& \nabla_{M} K_{N}=\frac{1}{18} e^{\frac{1}{2} a \phi}\left(f \epsilon_{M N M_{1} M_{2} M_{3}} H^{M_{1} M_{2} M_{3}}+3 H_{M N M_{3}} K^{M_{3}}\right), \\
& \nabla_{M} Y_{(3)}^{N_{1} N_{2}}=\frac{1}{18} e^{\frac{1}{2} a \phi}\left[6 Y_{(3) M M_{3}} H^{N_{1} N_{2} M_{3}}-6 \delta_{M}^{\left[N_{2}\right.} Y_{(3) M_{2} M_{3}} H^{\left.N_{1}\right] M_{2} M_{3}}\right. \\
& \left.-6 H_{M}{ }^{M_{3}\left[N_{2}\right.} Y_{(3)}^{\left.N_{1}\right]} M_{3}\right] \text {, } \\
& \nabla_{M} Y^{N_{1} N_{2}}=\frac{1}{18} e^{\frac{1}{2} a \phi}\left[6 Y_{M M_{3}} H^{N_{1} N_{2} M_{3}}-6 \delta_{M}^{\left[N_{2}\right.} Y_{M_{2} M_{3}} H^{\left.N_{1}\right] M_{2} M_{3}}\right.
\end{aligned}
$$




$$
\left.-6 H_{M}^{M_{3}\left[N_{2}\right.} Y^{\left.N_{1}\right]} M_{3}\right]
$$

It follows that $K \equiv K^{M} \partial_{M}$ is a Killing vector and

$$
\nabla_{[M} Y_{\left.(i) N_{1} N_{2}\right]}=0, \quad \nabla_{M} Y_{(i)}^{M N_{2}}=\frac{1}{3} e^{\frac{1}{2} a \phi} H_{(i) M_{2} M_{3}} F^{N_{2} M_{2} M_{3}}
$$

In addition, we have

$$
K^{M} \partial_{M} \phi=Y_{(i)} \wedge F=0, \quad Y_{(i)} \equiv Y_{(i) M N} d x^{M} \wedge d x^{N}
$$

With some lengthy algebra, we also obtain the following product relations

$$
\begin{aligned}
K_{M} K^{M} & =-f^{2}, \quad K_{N} Y_{(i)}^{N M}=0, \\
K_{\left[M_{1}\right.} Y_{\left.(i) M_{2} M_{3}\right]} & =-\frac{1}{6} \epsilon_{M_{1} M_{2} N_{1} N_{2} N_{3}} f Y_{(i)}^{M_{1} M_{2}}, \\
Y_{(i) N_{1}}{ }^{N_{3}} Y_{(j) N_{2} N_{3}} & =\delta_{i j}\left(f^{2} g_{N_{1} N_{2}}+K_{N_{1}} K_{N_{2}}\right)+\epsilon_{i j}{ }^{k} f Y_{(k) N_{1} N_{2}}, \\
Y_{(i)\left[N_{1} N_{2}\right.} Y_{\left.(j) N_{3} N_{4}\right]} & =\frac{1}{3} \delta_{i j} \epsilon_{M N_{1} N_{2} N_{3} N_{4}} f K^{M} .
\end{aligned}
$$

We are now in the position to derive the explicit solutions. For the case $f \neq 0$, the Killing vector $K$ is time-like and we can choose the coordinate such that $K=\partial_{t}$. Without loss of generality we consider $f>0$. We find that the general BPS solution is simply given by

$$
d s_{5}^{2}=-f^{2} d t^{2}+f^{-1} d s_{4}^{2}, \quad H_{(3)}=*_{4} d f^{-3}, \quad e^{a \phi}=f^{4},
$$

where the bi-spinor relations define the hyper-Kähler structure for $d s_{4}^{2}$. The Bianchi identity and the equation of motion for the 3 -form $H_{(3)}$ further imply that $f^{-3}$ is the harmonic function on the hyper-Kähler space $d s_{4}^{2}$.

When $f=0$, the Killing vector $K$ is null and we can choose the coordinate such that $K=\partial_{v}$. The bi-spinor relations and the Killing spinor equations imply that the general BPS solution is given by

$$
\begin{aligned}
& d s^{2}=H^{-1}\left(\lambda d u^{2}+2 d u d v\right)+H^{2}\left(d x_{i}+A_{i} d u\right)\left(d x^{i}+A^{i} d u\right), \\
& H_{(3)}=-3 H^{-4} \partial_{i} H d x^{i} \wedge d u \wedge d v-H^{-2} \partial_{i} A_{j} d x^{i} \wedge d x^{j} \wedge d u, \\
& e^{a \phi}=H^{4} .
\end{aligned}
$$

Note that there should be no confusion between the the 3 -form $H_{(3)}$ and the function $H$. After imposing the Bianchi identity and the equation of motion for $H_{(3)}$ as well as the Einstein equation along the $u$ direction, we find

$$
\partial_{i} \partial^{i} H^{3}=0, \quad \partial_{i} \partial^{i} A_{j}=0, \quad \partial_{[i} H \partial_{j} A_{k]}=0, \quad \partial_{i} \partial^{i} \lambda=-2 H^{3} \partial_{i} A_{j} \partial^{i} A^{j},
$$

where we have imposed the gauge condition $\partial_{u} H^{3}=\partial_{j}\left(H^{3} A^{j}\right)$ on $A_{j}$. 


\section{Conclusions}

In this paper we extend the construction of the Killing spinors in 2, 3] for non supersymmetric theories, by introducing scalar potentials. In the case of the effective action of the bosonic string, we find that the existence of consistent Killing spinor equations requires that the scalar potential is precisely the conformal anomaly term, which is a single exponential of the dilaton field. We obtain the most general tree-level action for the bosonic string up to the $\alpha^{\prime}$ order, suggesting that the hidden pseudo-supersymmetry associated with the Killing spinor equations is an intrinsic stringy property, regardless whether the theory is supersymmetric or not.

We also consider the Killing spinor equations for the Kaluza-Klein theories with scalar potentials in general dimensions. If the Killing spinor is neutral under the Kaluza-Klein vector, the scalar potential also has to be a single exponential term, which has no fixed point. However, we can gauge the theory such that the Killing spinors are charged under the $U(1)$ Kaluza-Klein vector, and the resulting scalar potential has one maximum, giving rise to an AdS vacuum. We obtain the charged black hole and discuss its BPS limit. The gauegd Kaluza-Klein theory has obvious application in the AdS/CFT correspondence. For $D=4,5,6$ and 7, these theories can be embedded in gauged supergravities and lifted to higher dimensional fundamental theories, such as M-theory or the type IIB theory. However, the origin of theories in general dimensions are not clear. The existence of Killing spinor equations warrants further investigations in these gauged Kaluza-Klein AdS theories.

Although in scalar-gravity theories, Killing spinor equations can be defined for an arbitrary superpotential, the situation is much more restrictive when form fields are involved. So far, the bosonic string and the Kaluza-Klein theory (22) with the scalar potential (37) are the only known non-trivial examples of intrinsically non-supersymmetry theories that admit Killing spinor equations. It is of great interest to investigate systematically the conditions for which Killing spinor equations can arise.

Finally we used the Killing spinor equations to obtain the most general solutions that admits at least one Killing spinor for the effective action for the five-dimensional bosonic string.

\section{Acknowledgement}

We are grateful to Chris Pope for useful discussions. H. Liu is grateful to KITPC, Beijing, for hospitality during the course of this work, and is supported in part by the National 
Science Foundation of China $(10425525,10875103)$, National Basic Research Program of China (2010CB833000) and Zhejiang University Group Funding (2009QNA3015).

\section{A KK reduction of Killing spinor equations}

Let us consider $(D+1)$-dimensional Einstein gravity

$$
\mathcal{L}_{D+1}=\sqrt{-\hat{g}} \hat{R}
$$

It's Killing spinor is defined by

$$
D_{M} \hat{\epsilon} \equiv \partial_{M} \hat{\epsilon}+\frac{1}{4} \omega^{A B}{ }_{M} \hat{\Gamma}_{A B} \hat{\epsilon}=0 .
$$

Performing the $S^{1}$ reduction,

$$
\begin{aligned}
d s_{D+1}^{2} & =e^{2 \alpha \phi} d s_{D}^{2}+e^{2 \beta \phi}(d z+A)^{2} \\
\beta & =-(D-2) \alpha, \quad \alpha^{2}=\frac{1}{2(D-1)(D-2)}
\end{aligned}
$$

the lower-dimensional Lagrangian is

$$
\mathcal{L}_{D}=\sqrt{-g}\left(R-\frac{1}{2}(\partial \phi)^{2}-\frac{1}{4} e^{a \phi} F_{(2)}^{2}\right)
$$

where $a=-2(D-1) \alpha$.

A convenient choice for the vielbein for the metric in (61) is given by

$$
\begin{aligned}
& \hat{e}^{A}{ }_{M}=e^{\alpha \phi} e^{A}{ }_{M}, \hat{e}^{Z}{ }_{M}=e^{\beta \phi} A_{M}, \\
& \hat{e}_{z}^{A}=0, \quad \hat{e}_{z}^{Z}{ }_{z}=e^{\beta \phi} .
\end{aligned}
$$

Note that $Z$ denotes the flat index. The spin connection is given by

$$
\begin{aligned}
\hat{\omega}^{A B} & =\omega^{A B}+\alpha e^{-\alpha \phi}\left(\partial^{B} \phi \hat{e}^{A}-\partial^{A} \phi \hat{e}^{B}\right)-\frac{1}{2} F^{A B} e^{(\beta-2 \alpha) \phi} \hat{e}^{Z}, \\
\hat{\omega}^{A Z} & =-\beta e^{-\alpha \phi} \partial^{A} \phi \hat{e}^{Z}-\frac{1}{2} F^{A}{ }_{B} e^{(\beta-2 \alpha) \phi} \hat{e}^{B} .
\end{aligned}
$$

Let us first consider the case when $D$ is even. The $(D+1)$-dimensional gamma matrices can be decomposed as

$$
\hat{\Gamma}_{A}=\Gamma_{A}, \quad \hat{\Gamma}_{Z}=\gamma
$$

where $\gamma$, with $\gamma^{2}=1$, is the chiral operator that is anti-commuting with all gamma matrices. The Killing spinor is given by

$$
\hat{\epsilon}=e^{\frac{1}{2} \alpha \phi} \eta
$$


The reduction of $D_{z} \hat{\epsilon}=0$ gives rise to

$$
-\gamma \Gamma^{M} \partial_{M} \phi \eta+\frac{1}{4 \beta} e^{(\beta-\alpha) \phi} \Gamma^{M N} F_{M N} \eta=0
$$

The reduction of $D_{M} \hat{\epsilon}=0$ gives rise to

$$
D_{M} \eta+\frac{\gamma}{8(D-2)} e^{(\beta-\alpha) \phi}\left(\Gamma_{M} \Gamma^{M_{1} M_{2}}-2(D-2) \delta_{M}^{M_{1}} \Gamma^{M_{2}}\right) F_{M_{1} M_{2}} \eta=0 .
$$

We now consider adding a cosmological constant in $D+1$ dimensions, namely $\mathcal{L}_{\text {cosmo }}=$ $-(D-1)(D-2) \lambda^{2} \sqrt{-g}$, which generates a scalar potential in $D$ dimensions:

$$
\mathcal{L}_{\text {pot }}=-(D-1)(D-2) \lambda^{2} e^{2 \alpha \phi} .
$$

The Killing spinor equation in $D+1$ is modified as

$$
\hat{D}_{M} \hat{\epsilon}+\frac{1}{2} \lambda \hat{\Gamma}_{M} \hat{\epsilon}=0
$$

The reduced Killing spinor equations become

$$
-\gamma \Gamma^{M} \partial_{M} \phi \eta+\frac{1}{4 \beta} e^{(\beta-\alpha) \phi} \Gamma^{M N} F_{M N} \eta-\frac{\lambda}{\beta} e^{\alpha \phi} \gamma \eta=0 .
$$

and

$$
\begin{aligned}
D_{M} \eta+ & \frac{\gamma}{8(D-2)} e^{(\beta-\alpha) \phi}\left(\Gamma_{M} \Gamma^{M_{1} M_{2}}-2(D-2) \delta_{M}^{M_{1}} \Gamma^{M_{2}}\right) F_{M_{1} M_{2}} \eta \\
& +\frac{D-1}{2(D-2)} \lambda e^{\alpha \phi} \Gamma_{M} \eta=0 .
\end{aligned}
$$

This implies that

$$
W=\sqrt{2}(D-1) \lambda e^{\alpha \phi} .
$$

Note that this scalar potential is different from given in section 4 .

We now consider the case when $D$ is odd. The gamma matrix decomposition is given by

$$
\hat{\Gamma}_{M}=\sigma_{1} \otimes \Gamma_{M}, \quad \hat{\Gamma}_{Z}=\sigma_{2} \otimes \mathbb{1}
$$

The reduced Killing spinor equations become

$$
\begin{aligned}
& D_{M} \eta-\frac{\mathrm{i}}{8(D-2)} e^{\frac{1}{2} a \phi} \sigma_{3}\left(\Gamma_{M} \Gamma^{M_{1} M_{2}}-2(D-2) \delta_{M}^{M_{1}} \Gamma^{M_{2}}\right) F_{M_{1} M_{2}} \eta \\
& \quad+\frac{W}{2 \sqrt{2}(D-2)} \sigma_{1} \Gamma_{M} \eta=0, \\
& \Gamma^{M} \partial_{M} \phi \eta-\frac{\mathrm{i}}{4} a e^{\frac{1}{2} a \phi} \sigma_{3} \Gamma^{M_{1} M_{2}} F_{M_{1} M_{2}} \eta-\sqrt{2} \frac{d W}{d \phi} \sigma_{1} \eta=0,
\end{aligned}
$$

where $W$ takes the same form as that in (173). Note that the Pauli matrices tensor product with the gamma matrices. The appearance of these pauli matrices reflects that the Killing 
spinors are symplectic Majorana. Not also that since $D$ is odd, so $(D+1)$ is even. This implies that in $(D+1)$ dimensions, the Killing spinor equation can have an alternative form $\hat{D}_{M} \hat{\epsilon}+\mathrm{i} \hat{\gamma} \hat{\Gamma}_{M} \hat{\epsilon}=0$. Since $\hat{\gamma} \sim \sigma_{3}$, thus it is equivalent to set $\sigma_{1}$ in the above equation to $\sigma_{2}$.

The reason we present the Kaluza-Klein reduction of pure gravity and its Killing spinor equations is to make comparison to the discussion in section 4. The abstract construction of the Killing spinors in the Kaluza-Klein theory is somewhat different from the dimensional reduction of the Killing spinors in one dimension higher. The integrability conditions suggest that they are equivalent. However, when the scalar potentials are added, they becomes inequivalent, each admits a different scalar potential. In particular, the Killing spinor

equations obtained from the Kaluza-Klein reduction cannot be gauged and $W$ cannot be augmented with an extra term to generate AdS spacetimes. This leaves the origin of the scalar potential (37) intriguing to investigate.

\section{References}

[1] D.Z. Freedman, C. Nunez, M. Schnabl and K. Skenderis, Fake supergravity and domain wall stability, Phys. Rev. D 69, 104027 (2004) arXiv:hep-th/0312055.

[2] H. Lü, C.N. Pope and Z.L. Wang, Pseudo-supersymmetry, consistent sphere reduction and Killing spinors for the bosonic string, arXiv:1105.6114 [hep-th], to appear in JHEP.

[3] H. Lü and Z.L. Wang, Killing spinors for the bosonic string, arXiv:1106.1664 [hep-th].

[4] H. Lü and Z.L. Wang, Pseudo-Killing spinors, pseudo-supersymmetric p-branes, bubbling and less-bubbling AdS spaces, to appear in JHEP, arXiv:1103.0563 [hep-th].

[5] M. Cvetič, H. Lü and C.N. Pope, Consistent Kaluza-Klein sphere reductions, Phys. Rev. D 62, 064028 (2000) arXiv:hep-th/0003286].

[6] S. Cucu, H. Lü and J.F. Vazquez-Poritz, Interpolating from $A d S_{D-2} \times S^{2}$ to $A d S_{D}$, Nucl. Phys. B 677, 181 (2004) arXiv:hep-th/0304022.

[7] H. Lü, C.N. Pope and J.F. Vazquez-Poritz, From AdS black holes to supersymmetric flux-branes, Nucl. Phys. B 709, 47 (2005) arXiv:hep-th/0307001].

[8] J. Perz, P. Smyth, T. Van Riet and B. Vercnocke, First-order flow equations for extremal and non-extremal black holes, JHEP 0903, 150 (2009) arXiv:0810.1528 [hepth]]. 
[9] J. Grover, J.B. Gutowski, C.A.R. Herdeiro and W. Sabra, HKT Geometry and de Sitter Supergravity, Nucl. Phys. B 809, 406 (2009) [arXiv:0806.2626 [hep-th]].

[10] J. Grover, J.B. Gutowski, C.A.R. Herdeiro, P. Meessen, A. Palomo-Lozano and W.A. Sabra, Gauduchon-Tod structures, Sim holonomy and De Sitter supergravity, JHEP 0907, 069 (2009) arXiv:0905.3047 [hep-th]].

[11] K. Skenderis and P. K. Townsend, Pseudo-supersymmetry and the domain-wall/cosmology correspondence, J. Phys. A 40, 6733 (2007) arXiv:hep-th/0610253.

[12] C.G. Callan, E.J. Martinec, M.J. Perry and D. Friedan, Strings in Background Fields, Nucl. Phys. B 262, 593 (1985).

[13] M. Cvetič, M.J. Duff, P. Hoxha, James T. Liu, H. Lü, J.X. Lü, R. Martinez-Acosta, C.N. Pope, H. Sati, T.A. Tran, Embedding AdS black holes in ten-dimensions and eleven-dimensions, Nucl. Phys. B 558, 96 (1999) arXiv:hep-th/9903214.

[14] L.J. Romans, The $F_{4}$ gauged supergravity in six dimensions, Nucl. Phys. B 269, 691 (1986).

[15] M. Cvetič, S.S. Gubser, H. Lü and C.N. Pope, Symmetric potentials of gauged supergravities in diverse dimensions and Coulomb branch of gauge theories, Phys. Rev. D 62, 086003 (2000) arXiv:hep-th/9909121.

[16] Z.W. Chong, H. Lü and C.N. Pope, BPS geometries and AdS bubbles, Phys. Lett. B 614, 96 (2005) arXiv:hep-th/0412221.

[17] J.P. Gauntlett, J.B. Gutowski, C.M. Hull, S. Pakis and H.S. Reall, All supersymmetric solutions of minimal supergravity in five- dimensions, Class. Quant. Grav. 20, 4587 (2003) arXiv:hep-th/0209114.

[18] S.Q. Wu, General rotating charged Kaluza-Klein AdS black holes in higher dimensions, Phys. Rev. D 83 (2011) 121502 (R) 